\title{
Evaluación de la Asimetría Vertical Mandibular, en Pacientes con Mordida Cruzada Posterior Uni y Bilateral
}

\author{
Evaluation of Mandibular Asymmetry in Unilateral and Bilateral Posterior Crossbite Patients
}

\author{
Claudia S. Arenas; Pamela Araya-Díaz \& Hernán Palomino M.
}

\begin{abstract}
ARENAS, C. S.; ARAYA-DÍAZ, P. \& PALOMINO, M. H. Evaluación de la asimetría vertical mandibular, en pacientes con mordida cruzada posterior uni y bilateral. Int. J. Morphol., 30(3):883-890, 2012.

RESUMEN: El objetivo fue determinar la existencia de asimetría vertical mandibular, en pacientes con mordida cruzada posterior uni y bilateral, tanto en dentición mixta primera fase como en permanente completa. Se recolectaron radiografías panorámicas correspondientes a 121 pacientes con dentición mixta primera fase y permanente completa, 57 de los cuales, presentaba mordida cruzada unilateral, 21 mordida cruzada bilateral y 43 individuos con oclusión normal, que sirvieron como grupo control. En las radiografías, se cuantificaron medidas lineales de altura condilar, coronoidea, de rama, cóndilo-más-rama y la diferencia entre las alturas de cóndilo y coronoides. Además, se calcularon los índices de asimetría para cada una de dichas estructuras. Se determinó la existencia de diferencias significativas entre los grupos, mediante el cálculo de los test Mann-Whitney y Kruskal-Wallis, dada la distribución de las variables y un test de chi cuadrado para evaluar asociación entre las mismas, con un intervalo de confianza de $95 \%$ ( $p<, 05$ ). Existen diferencias significativas entre los índices de rama y de cóndilo-más-rama, entre el grupo control y el grupo con mordida cruzada unilateral (MCU), al igual que en el índice coronoideo, entre el grupo control y el grupo con mordida cruzada bilateral. El grupo con MCU, presentó un mayor porcentaje de pacientes con asimetría de cóndilo y rama, encontrándose una leve asociación entre la condición de mordida cruzada unilateral y la asimetría de dichas estructuras. Dados los resultados, se puede concluir que existe asimetría en el cóndilo, coronoides y en cóndilo-menoscoronoides y coronoides, tanto en pacientes con mordida cruzada uni como bilateral. Sin embargo, el grupo con MCU presenta un mayor porcentaje de pacientes con asimetría de cóndilo y rama, existiendo una leve asociación entre MCU y asimetría de dichas estructuras.
\end{abstract}

PALABRAS CLAVE: Mordida cruzada posterior; Asimetría condilar; Asimetría mandibular; Ortopantomografía.

\section{INTRODUCCIÓN}

Las maloclusiones son afecciones del desarrollo, que corresponden a desviaciones clínicamente significativas, en la normalidad de las relaciones espaciales, crecimiento y morfología entre las bases óseas maxilares, las piezas dentarias, y de éstas con los maxilares (Moyers, 1992). Así, las maloclusiones pueden ser displasias dentarias, esqueléticas y/o dentoesqueléticas, pudiendo además presentarse en los 3 sentidos del espacio: vertical, sagital y transversal (Vellini, 2004).

Dentro de las alteraciones en sentido transversal, se encuentran las mordidas cruzadas posteriores, en las cuales se observa que las cúspides vestibulares de los premolares y molares superiores, ocluyen en las fosas de los premolares y molares inferiores, de forma que los sectores laterales inferiores, desbordan lateralmente a los superiores (Canut, 2000).

Su etiología puede estar relacionada con alteraciones esqueléticas, dentoalveolares o componentes neuromusculares funcionales, pero, la causa más frecuente es la falta de desarrollo del maxilar, causada principalmente por malos hábitos como succión digital, hábitos de deglución y obstrucción de la vía aérea superior (Andrade et al., 2009; Kiki et al., 2007). En pacientes con mordida cruzada posterior bilateral, las cúspides vestibulares de los dientes superiores, ocluyen por lingual de las cúspides vestibulares de los dientes mandibulares correspondientes, en ambas hemiarcadas (Kiki et al.). En los pacientes con mordida cruzada unilateral, en cambio, esta relación oclusal alterada, afecta sólo a una hemiarcada (Uysal et al., 2009).

Los cóndilos, en el lado de la mordida cruzada, estarían posicionados más superior y posteriormente en la fosa mandibular, en comparación al lado no afectado. Sin embargo, dado que puede ocurrir una remodelación ósea en la articulación temporomandibular, con el tiempo los cóndilos se ubicarían más simétricamente en su fosa, pero la asimetría facial y la desviación de la línea media mandibular ha- 
cia el lado de la mordida cruzada, podría persistir. La actividad de la neuromusculatura, posterior a la posición mandibular adquirida, puede además provocar un crecimiento mandibular asimétrico y varios cambios funcionales en los músculos masticatorios (Andrade et al.). Un estudio anterior, en adolescentes, mostró una menor actividad muscular durante la máxima intercuspidación, al morder y masticar en el lado con mordida cruzada, en comparación con el lado sin mordida cruzada y los controles. Además, en estos pacientes, los músculos maseteros derecho e izquierdo y temporal, se contraen de forma diferente y asimétrica (Uysal et al.).

Dado que las regiones de la mandíbula que tienen un mayor potencial de crecimiento, son los cartílagos condilares, alteraciones que ocurran en estas áreas pueden alterar su potencial de crecimiento, dando por resultado un desplazamiento de la mandíbula hacia el lado afectado. Es así como la asimetría de cóndilo, podría ser una de las causas más importantes de la asimetría mandibulofacial (Sezgin et al., 2007; Iturriaga et al., 2012).

Habets et al., (1988), investigaron la relación entre la asimetría condilar y los trastornos temporomandibulares, e introdujeron un método para determinar la asimetría entre los cóndilos y las ramas mandibulares, el cual compara la altura vertical del cóndilo y rama mandibular de los lados izquierdo y derecho, en radiografías panorámicas (Uysal et al.). Utilizando este método, Habets et al., (1988) elaboraron un índice de asimetría, en el que resultados mayores a $3 \%$ indicarían asimetría vertical, y valores menores, pueden deberse a errores técnicos durante la exposición de la película.

Este índice, fue utilizado para determinar si existe asimetría vertical mandibular, en pacientes con mordida cruzada uni y bilateral, en radiografías panorámicas.

\section{MATERIAL Y MÉTODO}

Se realizó un estudio descriptivo, de corte transversal, en sujetos en dentición mixta primera fase y permanente completa. Se seleccionaron 3 grupos de pacientes, procedentes de una clínica de práctica privada de ortodoncia y de la clínica de postgrado de Ortodoncia y Ortopedia Dento Máxilo Facial, de la Universidad Andrés Bello, Santiago de Chile.

El grupo control (GC), correspondió a 43 individuos diagnosticados con oclusión normal, es decir, relación canina y molar de neutroclusión, sin apiñamiento o apiñamiento leve y relación transversal intermaxilar normal. Los grupos de estudio estuvieron constituidos por 57 pacientes diagnosticados con mordida cruzada posterior unilateral (MCU), en al menos dos piezas dentarias, y 21 pacientes con mordida cruzada bilateral (MCB), en al menos 2 piezas dentarias en cada hemiarcadas. El diagnóstico se realizó, mediante un examen clínico, análisis de fotografías intra y extraorales y análisis de modelos de estudio. Los tres grupos presentaban dentición mixta primera fase (con incisivos completamente erupcionados), o dentición permanente completa, sin tratamiento previo.

Fueron eliminados de la muestra, aquellos pacientes con antecedentes médicos significativos, destrucción o caries extensa de piezas dentarias, historia previa de trauma facial, tratamiento ortodóncico o quirúrgico facial, malformaciones congénitas o adquiridas que afecten la región craneofacial y antecedentes de TTM.

La investigación se llevó a cabo utilizando radiografías panorámicas convencionales, las cuales fueron tomadas con la cabeza de los pacientes orientada en relación al plano horizontal de Frankfurt y los labios en reposo, tal como propone Azevedo et al., (2006). Las radiografías con evidente alteración de nitidez y/o calidad, fueron excluidas de este estudio.

Análisis radiográfico. Una vez obtenida la muestra, un examinador previamente entrenado y calibrado, trazó sobre papel de acetato, el contorno de la rama, el cóndilo y el margen inferior de la mandíbula, de ambos lados. Posteriormente, ubicó los puntos cefalométricos y trazó las medidas lineales.

Para la calibración, se realizó una repetición de las medidas, en 10 radiografías, en 2 días no consecutivos. El nivel de concordancia adquirido, entre ambas mediciones, se comprobó a través del cálculo del coeficiente de correlación intra-clase ( $\mathrm{ICC}=0,9)$.

El análisis cefalométrico utilizado para determinar la asimetría vertical mandibular, corresponde a una modificación del método propuesto por Habets et al., (1988) (Fig. 1).

Para la realización de esta investigación, el punto O1 no fue utilizado para la medición de la altura condilar $(\mathrm{CH})$, en cambio, como muestra la Figura 1, se utilizó la línea C: perpendicular a la línea A y tangente al punto más inferior de la incisura mandibular, la cual fue utilizada también por Kjellberg et al. (1995), en su estudio de asimetría mandibular. También se agregó el punto O3, correspondiente al punto más superior del proceso coronoides, y finalmente, se agregó la medición de la altura coronoidea $(\mathrm{CrH})$, que corresponde a la distancia entre $\mathrm{O} 3$ y línea $\mathrm{C}$. 


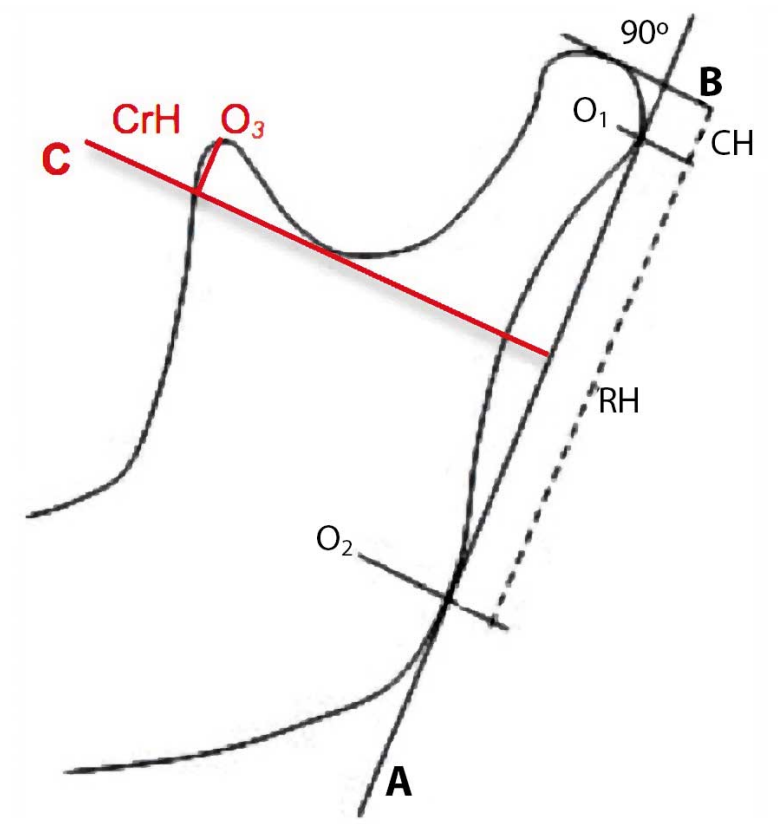

Fig. 1. Puntos utilizados por Habets en su modelo original y en rojo, aquellos puntos adicionalmente utilizados en el presente estudio. O1 : Punto más posterior del cóndilo; $\mathrm{O} 2$ : Punto más posterior de la rama; A: Tangente a los puntos $\mathrm{O} 1$ y $\mathrm{O} 2$; B: Línea perpendicular desde "A" al punto más superior del cóndilo; $\mathrm{CH}$ : Altura condilar; RH: Altura de la rama.

Análisis de los datos. Una vez obtenidos los datos, éstos fueron tabulados en formato Excel, se realizaron los análisis estadísticos con el software Prism 5 para MAC OSX.

1. Análisis descriptivo y comparación entre variables. Se calcularon los valores promedios y desviaciones estándar de cada variable, posteriormente, se determinó que la distribución de todas las variables no era normal, con el test de Kolmogorov-Smirnov y, en consecuencia, se utilizaron test no paramétricos

Inicialmente, se compararon los datos correspondientes a los lados derecho e izquierdo de cada paciente $(\mathrm{CH}$, $\mathrm{RH}, \mathrm{CrH}, \mathrm{CH}+\mathrm{RH}$ y $\mathrm{CH}-\mathrm{CrH}$ ), tanto en el grupo control (GC) como en el grupo de pacientes con MCB con un test de Mann-Whitney U.

Al comprobar la no existencia de diferencias significativas, se utilizó el conjunto de datos, sin distinguir lados derecho de izquierdo, en los GC y MCB.

Posteriormente se realizaron las siguientes comparaciones, utilizando el test Mann-Whitney U:

- Se comparó cada variable entre el lado afectado (con mordida cruzada) y no afectado del grupo con MCU.
- Cada variable del GC, con los datos correspondientes al lado afectado (con mordida cruzada) y el lado no afectado del MCU.

- Por último, las variables del GC se compararon con los datos obtenidos en el grupo de pacientes con MCB.

\section{2. Índices de asimetría.}

Para cada grupo, fueron calculados los índices de asimetría según las siguientes fórmulas propuestas por Habets et al.:

-Índice de asimetría condilar: (CHder-CHizq/ CHder+CHizq) $* 100$.

-Índice de asimetría de rama: (RHder-RHizq/ RHizq+RHder)*100.

-Índice de asimetría cóndilo-mas-rama: $[(\mathrm{CH}+\mathrm{RHder})$ $(\mathrm{CH}+\mathrm{RHizq}) /(\mathrm{CH}+\mathrm{RHder})+(\mathrm{CH}+\mathrm{RHizq})] * 100$.

Y los índices de asimetría creados para este estudio, según las siguientes fórmulas:

-Indice de asimetría coronoideo: (CrHder-CrHizq/ CrHder+CrHizq)*100.

-Indice de asimetría condilo-menos-coronoides: [ $(\mathrm{CH}-$ CrHder $)-(\mathrm{CH}-\mathrm{CrHizq}) /(\mathrm{CH}-\mathrm{CrHder})+(\mathrm{CH}-$ CrHizq) ]*100.

Si el valor de este índice era mayor a 3\%, se consideró la existencia de asimetría mandibular vertical, valores menores se atribuyeron a posibles variaciones en la técnica radiográfica (Habets et al., 1987).

Inicialmente, se compararon los índices obtenidos en los pacientes con dentición mixta y permanente para cada grupo (test Mann-Whitney U), y tras comprobar que no existían diferencias significativas en ninguno de los índices, se analizaron los datos de ambas denticiones en conjunto.

Luego, se analizó la existencia de diferencias significativas en los índices de asimetría, con un test ANOVA para variables no paramétricas (Kruskal-Wallis test) y un test Post-Hoc de Dunn para determinar la diferencia entre pares de grupos.

3. Asociación entre mordida cruzada y asimetría mandibular

Finalmente, se evaluó el grado de asociación entre asimetría y mordida cruzada posterior, tanto uni como bilateral, mediante el cálculo del test de chi cuadrado y Phi. 


\section{RESULTADOS}

Descripción de la muestra. De los 121 pacientes examinados, 43 presentaron oclusión normal ( 24 hombres, 19 mujeres), con una edad promedio de 13,9 $\pm 7,28 ; 57$ presentaron mordida cruzada unilateral (22 hombres, 35 mujeres) con una edad promedio de $13,7 \pm 10,44$; y 21 con mordida cruzada bilateral (12 hombres, 9 mujeres), con una edad promedio de $13,5 \pm 11,12$; no se encontraron diferencias estadísticamente significativas al comparar cada variable entre hombres y mujeres.

Análisis descriptivo y comparación entre variables. $\mathrm{Al}$ comparar las medidas lineales del grupo control con el lado afectado del grupo con $\mathrm{MCU}$, se observó que sólo la altura coronoidea presentaba diferencia significativa $(\mathrm{p}=0,040)$. Mientras que no se encontraron diferencias en ninguna de las variables al comparar el grupo control con el lado no afectado de este mismo grupo.

Tampoco se encontraron diferencias significativas en ninguna de las variables entre el GC y el grupo con MCB ni entre el lado afectado y no afectado del grupo con MCU.

Índices de asimetría (Tabla I, Fig. 2).

a) Índice Condilar: El índice condilar resultó ser, en promedio, mayor a $3 \%$ en todos los grupos, no existiendo diferencia significativa entre los grupos ni entre pares de grupos.

b) Índice de Rama: Se observó un índice promedio mayor al $3 \%$ en los grupos con MCU y MCB, no así en el grupo control, existiendo diferencia significativa entre los grupos $(\mathrm{p}=0,0048)$. El test de Dunn, señala que existe diferencia sólo entre GC y el grupo con MCU.

Tabla I. Comparación de los índices de asimetría entre los grupos control y con mordida cruzada uni y bilateral.

\begin{tabular}{llccc}
\hline & & Promedio & DS & $\boldsymbol{p}$ \\
\hline \multirow{3}{*}{ Índice Condilar } & Control & 3,09 & 2,7 & \\
& Unilateral & 4,84 & 3,6 & 0,0759 \\
& Bilateral & 4,45 & 3,28 & \\
Índice Rama & Control & 2,02 & 2,36 & \\
& Unilateral & 4,56 & 3,46 & 0,0048 \\
& Bilateral & 3,39 & 2,5 & \\
Índice Coronoideo & Control & 7,53 & 6,59 & \\
& Unilateral & 11,2 & 9,6 & 0,0296 \\
Índice Cóndilo-más-Rama & Bilateral & 14,81 & 8,72 & \\
& Control & 0,88 & 1,23 & \\
& Unilateral & 2,43 & 2,28 & 0,0220 \\
Índice Cóndilo-menos- & Bilateral & 2,14 & 2,39 & \\
Coronoides & Control & 7,13 & 5,22 & \\
& Unilateral & 11,22 & 12,9 & 0,0941 \\
& Bilateral & 15,21 & 13,53 & \\
\hline
\end{tabular}

c) Índice Coronoídeo: Superó el 3\% en todos los grupos, existiendo diferencia significativa entre ello $(p=0,0296)$, El test de Dunn señala que ésta diferencia existe sólo entre el grupo control y el grupo con MCB.

d) Índice Cóndilo-mas-Rama: resultó ser inferior a 3\% en todos los grupos, existiendo diferencia significativa entre ellos $(\mathrm{p}=0,022)$. El test de Dunn, señala que ésta diferencia se produce sólo entre el GC y con MCU.

d) Índice Cóndilo-menos-Coronoides: supera el 3\% tanto en el grupo control como en los grupos con MCU y MCB, no existiendo diferencia significativa entre ellos $(\mathrm{p}=0,0941)$.

En la Figura 2, se muestran los índices de asimetría promedio, de cada grupo. Se observa que en los tres grupos, el índice cóndilo-más-rama es menor a 3\% (sin asimetría) y que los índices coronoideo y cóndilo-menos-coronoides presentan los mayores valores de asimetría. (IC: Índice condilar, IR: Índice de rama, ICr: Índice coronoideo, IC+R: Índice cóndilo-más-rama $(\mathrm{CH}+\mathrm{RH})$

En cuanto a la cantidad de pacientes que presentan asimetría (Tabla II), se observa que el $66,7 \%$ de pacientes con MCU, presenta asimetría condilar versus un $44,1 \%$ en el GC, $64,9 \%$ asimetría de rama versus un $37,2 \%$ en el GC, $84,2 \%$ asimetría de coronoides versus un $69,7 \%, 28,1 \%$ asimetría de cóndilo-más-rama versus un $14 \%$ y $80,7 \%$ asimetría de cóndilo-menos-coronoides, versus un $81,3 \%$.

En los pacientes con MCB, 66,7\% presenta asimetría condilar, 52,4\% asimetría de rama, 90,5\% asimetría de coronoides, 23,8\% asimetría de cóndilo-másrama y $95,2 \%$ asimetría de cóndilo-menoscoronoides.

Asociación entre mordida cruzada y asimetría mandibular

$\mathrm{Al}$ analizar los resultados del test $\mathrm{Chi}$ cuadrado y Phi, se obtuvo que sólo hay una leve asociación entre mordida cruzada unilateral y asimetría condilar $\left(\chi^{2}: 0,025, \mathrm{p}<0,05\right.$; phi: 0,225$)$ y de rama $\left(\chi^{2}: 0,006, p<0,05\right.$; phi: $0,275)$. 


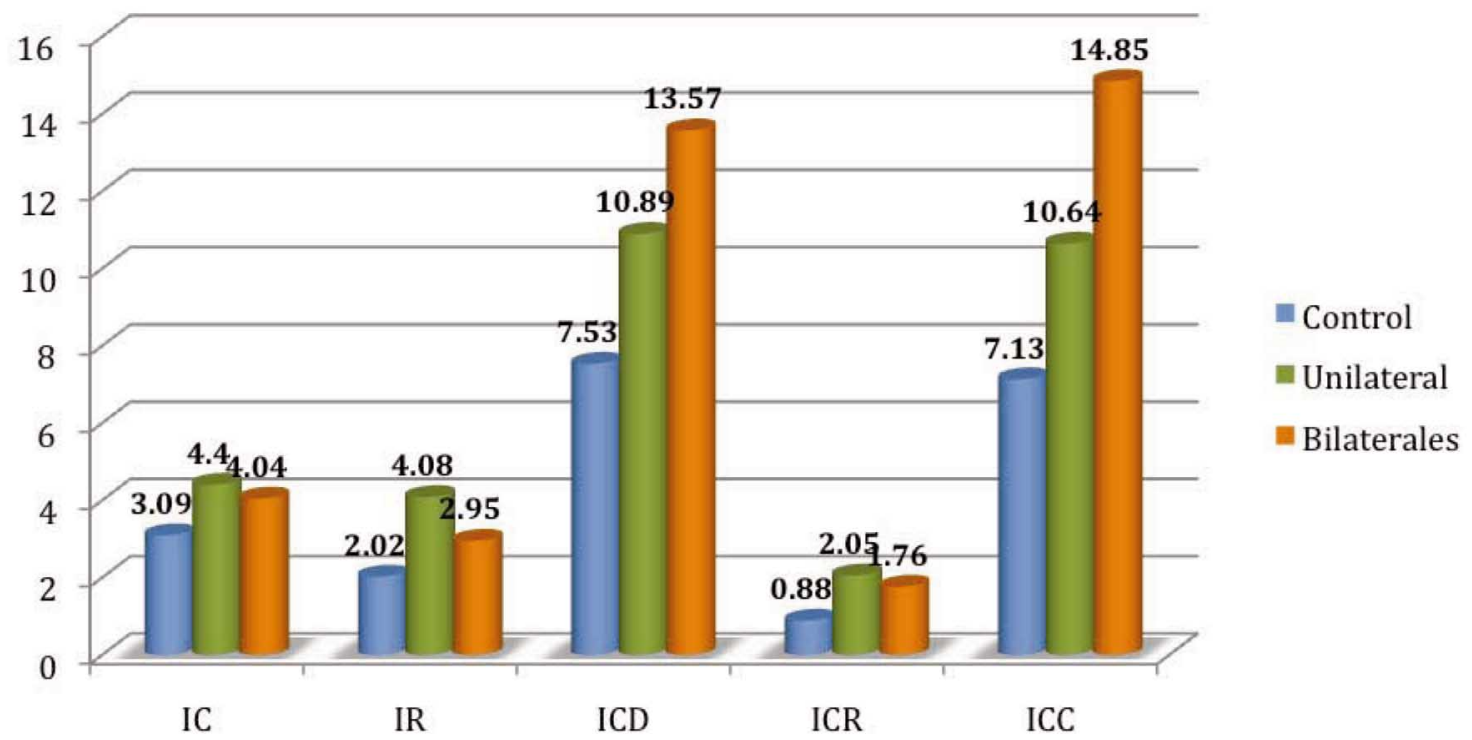

Fig. 2. Índices de asimetría del grupo control y grupos de estudio.

Tabla II. Cantidad de pacientes con y sin asimetría en los grupos control y con mordida cruzada uni y bilateral

\begin{tabular}{lcccccc}
\hline ÍNDICES & \multicolumn{3}{c}{ CONDILAR } & \multicolumn{3}{c}{ RAMA } \\
\hline OCLUSIÓN & Asimetría & Nomal & Total & Asimetría & Normal & Total \\
\hline Bilateral & 14 & 7 & 21 & 11 & 10 & 21 \\
Normal & 19 & 24 & 43 & 16 & 27 & 43 \\
Unilateral & 38 & 19 & 57 & 37 & 20 & 57 \\
Total & 71 & 50 & 121 & 64 & 57 & 121 \\
\hline ÍNDICES & \multicolumn{2}{c}{ CONDILO/RAMA } & & CONDILO/CORONOIDES \\
\hline OCLUSIÓN & Asimetría & Normal & Total & Asimetría & Normal & Total \\
\hline Bilateral & 5 & 16 & 21 & 20 & 1 & 21 \\
Normal & 6 & 37 & 43 & 35 & 8 & 43 \\
Unilateral & 16 & 41 & 57 & 46 & 11 & 57 \\
Total & 27 & 94 & 121 & 101 & 20 & 121 \\
\hline
\end{tabular}

\begin{tabular}{lccc}
\hline ÍNDICES & \multicolumn{3}{c}{ CORONOIDEO } \\
\hline OCLUSIÓN & Asimetría & Nomal & Total \\
\hline Bilateral & 19 & 2 & 21 \\
Normal & 30 & 13 & 43 \\
Unilateral & 48 & 9 & 57 \\
Total & 97 & 24 & 121 \\
\hline
\end{tabular}

\section{DISCUSIÓN}

En el presente estudio, se utilizaron radiografías panorámicas para evaluar asimetría vertical en la mandíbula. El uso de éste tipo de radiografías, ha sido aceptado para realizar mediciones en sentido vertical, aún cuando presentan distorsiones atribuibles a la propia técnica radiográfica
(Iturriaga et al.). Es así como, en un estudio, Kambylafkas et al., (2006) estableció, mediante la comparación de medidas directas sobre cráneos y radiografías panorámicas, que éstas sí pueden ser utilizadas para determinar asimetría vertical mandibular.

Al comparar las medidas lineales correspondientes a la altura condilar $(\mathrm{CH})$, altura de la rama $(\mathrm{RH})$, altura de cóndilo-másrama $(\mathrm{CH}+\mathrm{RH})$, altura de coronoides $(\mathrm{CrH})$ y cóndilo-menos-coronoides ( $\mathrm{CH}-\mathrm{CrH})$, entre el lado derecho e izquierdo del GC y el grupo de pacientes con MCB, no se encontraron diferencias significativas, lo que permitió utilizar los datos de ambos grupos sin diferenciar lado izquierdo y derecho. No se realizó esta comparación en el grupo de pacientes con MCU, dado que tanto el lado derecho como el izquierdo, podrían tener o no la condición de mordida cruzada. Es así como se compararon los lados afectado y no afectado de éste grupo, no encontrándose diferencia significativa. Este resultado concuerda con lo encontrado por Uysal et al., en un estudio en que utilizó el mismo método de medición en 46 pacientes con un promedio de edad de 13,06 años, con mordida cruzada unilateral. Sin embargo, las medidas $\mathrm{CrH}$ y $\mathrm{CH}-\mathrm{CrH}$ creadas especialmente para este estudio, no pudieron ser comparadas con otros resultados, ya que no existen investigaciones en que éstas hayan sido utilizadas. 
Al comparar el GC con el lado no afectado del grupo con MCU, no se encontraron diferencias significativas, sin embargo, si hubo diferencias significativas en la altura coronoídea $(\mathrm{CrH})$ al compararlo con el lado afectado. Esto último, podría deberse a que la coronoides es la estructura de inserción del músculo temporal, el cual presentaría contracciones asimétricas en pacientes con MCU, según lo observado por Ben-Bassat et al. (1993) y Brin et al. (1996), al estudiar los aspectos funcionales y esqueletales de pacientes en dentición mixta con mordida cruzada unilateral, lo cual podría influenciar el crecimiento y por ende, las dimensiones de esta estructura anatómica.

Por otra parte, no se encontró diferencia significativa al comparar el GC con el grupo con MCB, en ninguna de las variables estudiadas. Estos resultados indicarían, que la morfología de la rama, cóndilo y coronoides en el lado no afectado del grupo con MCU y en los pacientes con MCB, se encuentran dentro de los rangos de normalidad y por lo tanto no habrían sido afectados por la maloclusión.

Posteriormente, para calcular los índices de asimetría, se utilizó el método de Habets et al. (1988), antes descrito, para evaluar asimetría mandibular en pacientes con trastornos temporomandibulares (Habets et al., 1988; Saglam \& Sanli, 2004) y varios tipos de maloclusión (Sezgin et al.; Kilic et al., 2008). De acuerdo con Habets et al. (1988), un valor de índice de $3 \%$ puede ser resultado de un cambio de $1 \mathrm{~cm}$ en la posición de la cabeza, durante la toma radiográfica y por lo tanto, sólo los valores mayores a 3\% se consideraron como asimetría vertical mandibular.

Aunque las medidas lineales de altura de cóndilo, rama, cóndilo-más-rama, coronoides y cóndilo-menoscoronoides, fueron similares entre el lado derecho e izquierdo en el GC, se encontró una leve asimetría condilar en los individuos del grupo con oclusión normal (3,5\%), lo que concuerda con lo encontrado por Uysal et al. (7,57\%), Kilic et al. $(3,81 \%)$ y Saglam (2003), en los individuos con clase I dentaria y esqueletal $(7,96 \%)$. Sin embargo, tampoco se ha encontrado asimetría condilar en los grupos control de algunos estudios (Uysal et al.; Habets et al., 1988; Miller \& Smidt, 1996), lo cual podría deberse a que los rangos de edad de los pacientes estudiados, difieren de los utilizados en el presente estudio. También se encontró que los índices de asimetría para la coronoides y para cóndilocoronoides, fueron mayores a $3 \%$ en este grupo (GC). Al analizar éste resultado, se debe considerar que la coronoides es la estructura ubicada más cercana a la línea media en la radiografía, por lo que está sujeta a mayores distorsiones en la técnica radiográfica. Nuevamente, estos parámetros (índices $\mathrm{CrH}$ y $\mathrm{CH}-\mathrm{CrH}$ ) no pudieron ser comparados con otros estudios.
El índice condilar presentan asimetría en ambos grupos de estudio. Además, para este índice, se encontró diferencias significativas entre el grupo control y el grupo con $\mathrm{MCU}$, al igual que lo encontrado por Kilic et al. e Inui et al. (1999), sugirieron que el desplazamiento contínuo del cóndilo en la cavidad glenoidea, durante el período de crecimiento, derivado de los problemas oclusales, induciría diferencias en el crecimiento de los cóndilos derecho e izquierdo. Desde esta perspectiva, estas asimetrías podrían ser el resultado de una desviación funcional repetitiva de la mandíbula en los sujetos con mordida cruzada.

Al comparar el índice condilar del GC con el grupo con $\mathrm{MCB}$, no se encontraron diferencias significativas, al igual que en el estudio de Uysal et al. sin embargo, Kiki et al. si encontraron diferencias estadísticamente significativas, lo cual podría deberse a la diferencia en el tamaño de la muestra, ya que para este estudio se estudiaron las radiografías de 21 pacientes con esta condición, en contraposición con las 75 utilizadas por Kiki et al. en su estudio. Este autor explica que el Indice Condilar más alto, se debería a la falta de armonía interoclusal en los pacientes con mordida cruzada.

El índice de asimetría de rama (IR), fue mayor a 3\% en el grupo con MCU y MCB, al igual que lo encontrado por Uysal et al. (3,03\%). Además, al comparar MCU con el IR del grupo control, se encontraron diferencias significativas.

El crecimiento de la rama mandibular, está influenciado por las fuerzas funcionales del músculo masétero, el cual se contrae de forma asimétrica en los pacientes con mordida cruzada unilateral, al igual que los demás músculos masticatorios, según lo describió Ben-Bassat et al. y Brin et al. al evaluar el patrón masticatorio de estos pacientes, mediante electromagnetismo, lo que podría explicar la diferencia en el crecimiento de ambos lados. Sin embargo, Uysal et al., no obtuvo diferencias significativas al comparar el IR entre el grupo control y el grupo con MCU.

El índice $\mathrm{CH}+\mathrm{RH}$, fue menor a $3 \%$ en los tres grupos, al igual que lo encontrado por Uysal et al. Kiki et al. y Kilic et al.

Los índices coronoideo y cóndilo-menos-coronoides (IC-Cr) arrojaron una elevada asimetría en los tres grupos, no encontrándose diferencia significativa.

Este nivel tan elevado de asimetría, puede ser explicado en parte, si consideramos que, como se mencionó anteriormente, el crecimiento de la coronoides se ve afectado por la actividad del músculo temporal, el cual en sujetos con mordida cruzada presenta una contracción asimétrica (Ben-Bassat et al.; Brin et al.). Además, es una estructura 
que está sujeta a mayor distorsión durante la toma de la radiografía panorámica. Sin embargo, aún cuando la morfología coronoidea sea variable y la distorsión que sufre durante la técnica radiográfica sea significativa, la asimetría resultante es tal, que pone en cuestionamiento la validez de estos índices (coronoideo e IC-Cr), por lo cual, su confiabilidad y utilidad clínica, deben ser evaluadas en el futuro.

Las diferencias entre los resultados encontrados al comparar las medidas lineales y los índices entre los grupos, podría explicarse dado que la comparación de medidas lineales se basa en promedios que pueden no representar la situación de cada paciente en particular, y ser influenciado por la variabilidad en el tamaño de las estructuras entre un paciente y otro, mientras que los índices, son calculados para cada paciente en particular y luego sometidos al análisis inferencial, lo que de alguna manera elimina la variable tamaño y normaliza los datos, aumentando la confiabilidad de estos resultados.

Al evaluar el grado de asociación entre la condición de mordida cruzada y la asimetría mandibular, se encontró que los índices de asimetría de cóndilo (IC) y rama (IR) son los únicos que estarían levemente asociados, a la presencia de MCU. No se encontraron estudios previos que evaluaran dicha asociación.

Al analizar la diferencia de los índices entre dentición mixta primera fase y dentición permanente, si bien ésta no es significativa, se observó que la asimetría de la rama en los pacientes con MCU, aumenta a través del tiempo, ya que en dentición mixta el promedio del índice de rama es de $4,09 \%$, mientras que en dentición permanente es de 5,24\%, lo que podría indicar que al no tratarse la MCU a edades tempranas, la asimetría de esta estructura progresaría y se haría más evidente en la dentición permanente.

Podemos concluir que, se observó asimetría en el cóndilo, coronoides y en IC-Cr, en todos los grupos estudiados, mientras que la rama resultó ser asimétrica sólo en los pacientes con MCU y que el grupo con MCU, presenta mayor porcentaje de pacientes con asimetría de cóndilo y rama, existiendo una leve asociación entre MCU y asimetría de estas estructuras.

ARENAS, C. S.; ARAYA-DÍAZ, P. \& PALOMINO, M. H. Evaluation of mandibular asymmetry in unilateral and bilateral posterior crossbite patients. Int. J. Morphol., 30(3):883-890, 2012.

SUMMARY: The aim was to evaluate the mandibular vertical asymmetry, in unilateral and bilateral posterior crossbite patients both in permanent and mixed dentition. One hundred twenty one panoramic radiographs were collected, 57 from patients with unilateral posterior crossbite, 21 patients with bilateral posterior crossbite and 43 with normal occlusion that were used as a control group. Subsequently, reference points and cephalometric traces were drawn manually by a calibrated examiner in order to register the condylar, coronoideal, and ramal heigths. This data was used to calculate an asymmetry index for each structure. According to the distribution of the variables, Mann-Whitney and Kruskal-Wallis test was used to compare the data between groups of patients and chi square was used to evaluate association between them, at the $95 \%$ confidence interval $(\mathrm{p}<.05)$. Statistically significant differences were found in the ramal and condylar-plus-ramal asymmetry index values, between the control and the unilateral posterior crossbite group, also statistical differences were found in the coronoideal index values, between the control and the bilateral posterior crossbite groups. On the other hand, the bilateral crossbite group, presented a higher percentage of patients with condylar and ramal asymmetry, finding a mild statistically significant association between this condition and those with asymmetry. Condylar and coronoideal asymmetry was found both in the unilateral as in the unilateral posterior crossbite patients. The bilateral crossbite group, presented a major percentage of patients with asymmetry, with a mild association between those conditions.

KEY WORDS: Crossbite; Condylar asymmetry; Mandibular asymmetry; Orthopantomograph.

\section{REFERENCIAS BIBLIOGRÁFICAS}

Andrade, A. S.; Gameiro, G. H.; Derossi, M. \& Gavião, M. B. Posterior Crossbite and Functional Change. Angle Orthod., 79(2):380-6, 2009.

Azevedo, A. R.; Janson, G.; Henriquez, J. F. \& De Freitas, M. R. Evaluation of asymmetries between subjects with Class II subdivision and apparent facial asymmetry and those with normal occlusion. Am. J. Orthod. Dentofacial Orthop., 129(3):376-83, 2006.
Ben-Bassat, Y.; Yaffe, A.; Brin, I.; Freeman, J. \& Ehrlich, Y. Functional and morphological occlusal aspects in children treated for unilateral posterior crossbite. Eur. J. Orthod., 15(1):57-63, 1993.

Brin, I.; Ben-Bassat, Y.; Blustein, Y.; Ehrlich, J.; Hochman, N. \& Marmary, Y. Skeletal and functional effects of treatment for unilateral posterior crossbite. Am. J. Orthod. Dentofacial Orthop., 109(2):173-9, 1996. 
Canut, J. A. Ortopedia clínica y terapéutica, Capitulo 24: maloclusiones transversales. $2^{\mathrm{a}}$ ed. Barcelona, Ed. Salvat, 2000. pp.465-94.

Habets, L. L.; Bezuur, J. N.; Van Ooij, C. P. \& Hansson, T. L. The orthopantomogram, an aid in diagnosis of temporomandibular joint problems. I. The factor of vertical magnification. J. Oral Rehabil., 14(5):475-80, 1987.

Habets, L. L.; Bezuur, J. N.; Naeiji, M. \& Hansson, T. L. The Orthopantomogram, an aid in diagnosis of temporomandibular joint problems. II. The vertical symmetry. J. Oral Rehabil., 15(5):465-71, 1988.

Inui, M.; Fushima, K. \& Sato, S. Facial asymmetry in temporomandibular joint disorders. J. Oral Rehabil., 26(5):402-6, 1999.

Iturriaga, V.; Navarro, P.; Cantin, M. \& Fuentes, R. Prevalence of Vertical Condilar Asymmetry of the Temporomandibular Joint in Patients with Signs and Symptoms of Temporomandibular Disorders. Int. J. Morphol., 30(1):315-21, 2012.

Kambylafkas, P.; Murdock, E.; Gilda, E.; Tallents, R. H. \& Kyrkanides, S. Validity of panoramic radiographs for measuring mandibular asymmetry. Angle Orthod., 76(3):38893, 2006.

Kiki, A.; Kilic, N. \& Oktay, H. Condylar Asymmetry in Bilateral Posterior Crossbite Patients. Angle Orthod., 77(1):77-81, 2007.

Kilic, N.; Kiki, A. \& Oktay, H.. Condylar asymmetry in unilateral posterior crossbite patients. Am. J. Orthod. Dentofacial. Orthop., 133(3):382-7, 2008.

Kjellberg, H.; Fasth, A.; Kiliaridis, S.; Wenneberg, B. \& Thilander, B. Craniofacial structure in children with juvenile chronic arthritis (JCA) compared with healthy children with ideal or postnormal occlusion. Am. J. Orthod. Dentofacial Orthop., 107:67-78, 1995.

Miller, V. J. \& Smidt, A. Condylar asymmetry and age in patients with an Angle's Class II division 2 malocclusion. J. Oral Rehabil., 23(10):712-5, 1996.

Moyers, R. E. Manual de Ortodoncia, sección I: crecimiento y desarrollo, capítulo 7: etiología de la maloclusión. $4^{\mathrm{a}}$ ed. Buenos Aires, Médica Panamericana, 1992. pp.151-67.

Saglam, A. A. \& Sanli, G. Condylar asymmetry measurements in temporomandibular disorders. J. Contemp. Dent. Pract., 5(3):59-65, 2004.

Saglam, A. M. The condylar asymmetry measurements in different skeletal patterns. J. Oral Rehabil., 30(7):738-42, 2003.

Sezgin, O. S.; Celenkb, P. \& Aricic, S. Mandibular Asymmetry in Different Occlusion Patterns. Angle Orthod., 77(5):803-7, 2007.
Uysal, T.; Sisman, Y.; Kurt, G. \& Ramoglu, S. I. Condylar and ramal vertical asymmetry in unilateral and bilateral posterior crossbite patients and a normal occlusion simple. Am. J. Orthod. Dentofacial Orthop., 136(1):37-43, 2009.

Vellini, F. Ortodoncia, diagnóstico y panificación clínica, capitulo 5: clasificación de las maloclusiones. $2^{\mathrm{a}} \mathrm{ed}$. Sao Paulo, Artes Médicas Latinoamericana, 2004. pp.97-114.

Dirección para correspondencia:

Pamela Araya-Díaz

Departamento del Niño y Adolescente

Área de Ortodoncia

Facultad de Odontología

Universidad Andrés Bello

Santiago

CHILE

Email:payleen@gmail.com

Recibido : 26-01-2012

Aceptado: 29-06-2012 